\title{
Blood donors screening for malaria in non-endemic area in the Kingdom of Saudi Arabia: Is it necessary to introduce immunological testing?
}

\author{
Ghaleb Elyamany ${ }^{1,2}$, Ali Al Gharawi ${ }^{1}$, Mohammed Alrasheed ${ }^{1}$, Omar Alsuhaibani ${ }^{1}$
}

${ }^{1}$ Department of Central Military Laboratory and Blood Bank, Prince Sultan Military Medical City, Riyadh, Saudi Arabia

${ }^{2}$ Department of Hematology, Theodor Bilharz Research Institute, Egypt

\section{Type of article: Original}

\begin{abstract}
Introduction: In Saudi Arabia, where malaria is not endemic, the incidence is very low. However, malaria transmission cases have been reported, mainly in Asir and Jazan provinces along the Southwestern border with Yemen. Imported cases also have been reported. The aims of this study were to determine the prevalence of malaria in blood donors in a tertiary care hospital in the central area of Saudi Arabia and to assess the effectiveness of malaria screening methods used by transfusion services in Prince Sultan Military Medical City.

Methods: This study was conducted on 180,000 people who donated blood during 2006-2015. All blood smears from blood donors were screened for malaria infection using Giemsa staining, low power and high power microscopic examinations, and using oil immersion lens. The data were analyzed and reported in descriptive statistics and prevalence.

Results: From the total of 180,000 blood donors who were screened for malaria, 156,000 (87\%) and 23.400 (13\%) were Saudi Arabia citizens and non-Saudi residents, respectively. The mean age of the blood donors was 32 (ranging from 18 to 65 ), $97 \%$ and 3\% were male and female, respectively. Using our current method for malaria screening, the prevalence of malaria in the study population was zero.

Conclusion: The current methods of malaria screening in blood donors is not suitable for screening low-level parasiotemia. Adding the immunoassay and molecular screening methods is suggested.

Keywords: malaria, blood donors, peripheral blood smear, antibody testing
\end{abstract}

\section{Introduction}

The bite of female anopheles mosquitos transmits malaria to people. However, it also can be transmitted readily by blood transfusions using blood from collected from asymptomatic, parasitaemic donors (1). Transfusion transmitted malaria (TTM) can be acquired through blood components, such as platelets, leucocytes, fresh-frozen plasma, red cell concentrates, and frozen red blood cells (1-5). This is due to the fact that the parasites are stable in plasma and whole blood for at least 18 days when stored at $+4{ }^{\circ} \mathrm{C}$, and they are stable for more extended periods in the frozen state $(6,7)$. The World Health Organization's (WHO's) recommendation and the National Institutes of Health's (NIH's) Consensus Conference in 1995 requires that every donor who gives blood should be screened for various infections, including human immunodeficiency virus (HIV), Hepatitis B and C, malaria, and syphilis (8). However, there is no suitable test available for screening for malaria in the donors. The different diagnostic tests available for malaria include examination of a peripheral smear and various serological tests, including polymerase chain reaction (PCR). However, all of these tests have limitations in terms of specificity, sensitivity, and cost effectiveness. So, the challenge is to properly screen the donated blood for malaria by a test that should be sensitive, simple, fast, and economically feasible (9). The risk of TTM is complicated by the fact that infectious organisms can remain viable in the blood for a long, relatively-asymptomatic period of time. Falciparum malaria is common in the Kingdom of Saudi Arabia (KSA), and it can remain in the bloodstream for years while exhibiting no symptoms of infection (10).

\section{Corresponding author:}

Dr. Omar Alsuhaibani, Department of Central Military Laboratory and Blood Bank, Prince Sultan Military Medical City, Riyadh 11159, Saudi Arabia. Email: oalsuhaibani@yahoo.com

Received: December 04, 2015, Accepted: January 26, 2016, Published: February 2016

iThenticate screening: January 26, 2016, English editing: February 01, 2016, Quality control: February 04, 2016

(C) 2016 The Authors. This is an open access article under the terms of the Creative Commons Attribution-NonCommercialNoDerivs License, which permits use and distribution in any medium, provided the original work is properly cited, the use is non-commercial and no modifications or adaptations are made. 
Thus, most blood transfusion services have a policy of rejecting donors who may be at risk. Currently, many countries have legislation that requires a waiting period of six months to three years, depending on the estimated risk of exposure (5). Although such a deferral policy can be successful, the increase in the number of at-risk donors can cause shortages in blood stocks in many non-endemic countries. It is somewhat surprising that the value of screening for malaria using the current deferral policy has become controversial. It has been argued that the existing deferral criteria have been highly effective for ensuring that the blood supply is safe (11).

\section{Material and Methods}

In 2005, Prince Sultan Military Medical City, Saudi Arabia, started to include routine screening for malaria in the program of screening blood donors by examination for the malaria parasite. As many as 180,000 blood donors were screened for malaria during the period from June 2006 to June 2015. All donors fulfilled the criteria for blood donation, which included a clinical examination and an interview to record the history of previous infectious diseases, surgery, blood transfusion, heart diseases, anemia, and information on foreign travel. Examination for malaria parasite was done by thick and thin smear examination using standard methods (12). A thick smear stained with Giemsa stain and observed under a microscope at low power, high power, and then using oil immersion lens. If positive, a thin smear was made to identify the species.

\section{Results}

Over 180,000 blood donors' samples were screened for malaria infection. Of these, 156,600 donors were Saudi $(87 \%)$, and 23,400 were non-Saudi (13\%). The donors were 174,600 males (97\%) and 5,400 females (3\%), and their mean age was 32. (The ages ranged from 18 to 65.) All of the tested samples (Saudi and non-Saudi) were negative for malaria infection. The prevalence rate among blood donors was $0 \%$, since no positive cases were detected by thick blood films, which are the only screening test for blood donors except for a questionnaire that is used in our institute.

\section{Discussion}

Saudi Arabia is in the malaria elimination phase as classified by the World Health Organization (WHO), and the country reported just 34 local cases in 2013 (13). The malaria burden has been quite low since 2000 with the majority of cases, both local and imported, occurring in Asir and Jazan Provinces along the southwestern border with Yemen $(14,15)$. The main risk at the present time is the potential introduction of malaria parasites into the blood supply in KSA, and this risk is associated with semi-immune people. Despite the WHO's recommendation that donated blood be screened for malaria, this policy has been implemented by only a few blood centers. There are many reasons for this (16), the most important of which is that the only affordable method of blood screening was microscopic evaluation. This method is very labor intensive, and it takes at least 15-20 minutes of thick-film observation. This makes it unsuitable for use when there are large numbers of samples. In addition, this method is not very sensitive, and its results depend, to a significant extent, on the experience of the observer (17). Also, there are assay techniques available on the market that involve rapid tests using a dipstick, and there are microtiter plate enzyme immunoassays. Both HRP2 and PLDH are species specific, but the sensitivity is inadequate and the cost is high. At best, the level of detection of both microscopic evaluation and antigen detection is 100 parasites/ $\mu \mathrm{L}$ (18). Therefore, all screening assays are not considered to be sensitive enough to prevent malarial infections effectively. As a result of all these considerations, not much attention is paid to the safety of blood with respect to conveying malaria (17). Malaria transmission occurs in Saudi Arabia, and it is endemic mainly in the lowlands of the Asir region of the Southwestern Province (15), and imported cases have been reported (19-21). The prevalence of malaria infection is variable in different regions of the KSA, with the vast majority being generally very low since 2000. Most of the cases, both local and imported, occur in Asir and Jazan Provinces (22). In 2005, in Prince Sultan Military Medical City (PSMMC), KSA began to include routine screening for malaria in the blood donor screening program by examining samples for malaria parasites by thick and thin smear techniques using standard methods (12). The microscopic techniques lack the required sensitivity to detect a very low concentration of parasitaemia, such as 0.00004 parasite $/ \mu \mathrm{L}$. Although the sensitivity PCR tests has been reported to be as low as 0.004 parasite/ $\mu \mathrm{L}$, this is less than the required sensitivity (5). Cases of TTM with negative microscopic examination and positive high antibody titer have been recorded in the literature (23-27). Although there has been some debate over the most effective strategy for the prevention of TTM, donor deferral plus malarial antibody screening provides the most effective strategy in non-endemic countries (28-30). In our study, we used a large sample size of 180,000 from healthy blood donors in the central area of KSA. The prevalence rate was $0 \%$ since no positive cases were detected by thick blood films, which are the only screening test for blood donors other than a questionnaire used by our 
institute. The prevalence rate of malaria found among PSMMC blood donors was different than that observed in similar studies in the KSA $(21,31,32)$. At present, there are conflicting views about the need to add immunoassay and molecular screening tests for blood donors in non-endemic areas, such as the Middle East countries, including Saudi Arabia, because many of these countries still depend on screening donors using a questionnaire, and, in many countries, this is the only step in the prevention of TTM. Although the overall prevalence rate in the Kingdom is very low $(13,14)$ and additional screening does not appear to be cost effective, it is our opinion that the introduction of screening for malaria in the blood banks is appropriate in order to screen high risk groups and due to the presence of as many as two million expatriates in the Kingdom from different countries, including areas that are known to have a high prevalence of malaria. Although the results we reported do not show any significant difference in the prevalence of malaria between donors from Saudi Arabia and other donors, the fact that most of the non-Saudi donors come from malaria endemic areas seems to make it reasonable to conduct routine testing of these donors for malaria since they may have resided in areas where malaria is prevalent for a long time, allowing them to develop immunity. This recommendation is compatible with those of other researchers (19-22, 31). Another consideration is that there is increasing travel by the Saudi population into areas where malaria is prevalent, which means that the number of donors with potential malaria risk is likely to be increasing. Another concern is that blood donors may give inaccurate information because they misunderstand the questions or they are unaware that have had malaria (33). It may not be possible to prevent exposures to TTM completely (10). Any screening strategy that is used must focus on minimizing the risk of introducing malaria to the population without decreasing the availability of blood.

\section{Conclusions}

Currently, there is no sensitive, practical, and affordable method for screening blood for low-level parasitaemia that can be used by transfusion services. The most commonly used test is microscopy, but it is not sensitive enough and takes a lot of time. Although cases of TTM are not common in non-endemic countries, such as KSA, malaria is spreading, and the number of infected donors also is likely to be increasing. It is a matter of debate whether there is a need to require immunoassays and molecular screening tests for blood donors in non-endemic areas, such as Saudi Arabia. If immunological testing were to imposed nationwide as a valid method of assessing the risk of malaria, many of the currently-deferred donors could be accepted four months after their last visit to an area where malaria is known to be endemic, and this would increase the supply of blood.

\section{Acknowledgments:}

The authors thank the transfusion services staff at Prince Sultan Military Medical City for their contributions to this study.

\section{Conflict of Interest:}

There is no conflict of interest to be declared.

Authors' contributions:

All authors contributed to this project and article equally. All authors read and approved the final manuscript.

\section{References}

1) Woolsey G. Transfusion for pernicious anaemia: two cases. Ann Surg. 1911; 53: 132-5.

2) Garfield MD, Ershler WB, Maki DG. Malaria transmission by platelet concentrate transfusion. JAMA. 1978; 240: 2285-6. doi: 10.1001/jama.1978.03290210067033

3) Dover AS, Guinee VF. Malaria transmission by leukocyte component therapy. JAMA. 1971; 217: 1701-2. doi: $10.1001 /$ jama.1971.03190120067017.

4) Lazner E, Newhouser E. Studies on the transmissibility of malaria by blood transfusions. Am J Med Sci. 1943; 204: 141-6. doi: 10.1097/00000441-194308000-00001.

5) Grande R, Petrini G, Silvani I, Simoneschi B, Marconi M, Torresani E. Immunological testing for malaria and blood donor deferral: the experience of the $\mathrm{Ca}^{\prime}$ GrandaPolyclinic Hospital in Milan. Blood Transfus. 2011; 9(2): 162-6. PMID: 21251462, PMCID: PMC3096859.

6) World Health Organisation. World malaria report. Geneva: WHO; 2010.

7) Romualdo Grande, Gianna Petrini, Iris Silvani, Barbara Simoneschi, Maurizio Marconi, Erminio Torresani. Immunological testing for malaria and blood donor deferral: the experience of the $\mathrm{Ca}$ ' Granda Polyclinic Hospital in Milan. Blood Transfus. 2011; 9(2): 162-6. PMID: 21251462, PMCID: PMC3096859. 
8) Infectious disease testing for blood transfusions. NIH Consensus Panel on Infectious Disease Testing for blood transfusions. JAMA. 1995; 274(17): 1374-9. doi: 10.1001/jama.1995.03530170054032, PMID: 7563563.

9) Moiz B. Prevention of Transfusion Transmitted Malaria in an Endemic area- A Challenge for Blood Banks. Infectious Diseases Journal of Pakistan. 2004; 96-8.

10) Slinger R, Giulivi A, Bodie-Collins M, Hindieh F, John RS, Sher G, et al. Transfusion-transmitted malaria in Canada. CMAJ. 2001; 164: 377-9. PMID: 11232141 , PMCID: PMC80734.

11) Hanscheid T, Valadas E, Grobusch MP. Polymerase chain reaction for screening blood donors at risk for malaria: safe and useful? Emerg Infect Dis. 2002; 8(8): 872. doi: 10.3201/eid0808.020025, PMID: 12141979, PMCID: PMC2732529.

12) Chatterjee KD. Malarial parasites of man. In: Chatterjee KD, editor. Parasitology in relation to Clinical Medicine. 12th ed. Calcutta: Chatterjee Medical Publishers; 1995: 215-6

13) Global Malaria Programme. World Malaria Report 2014. Geneva: World Health Organization; 2014.

14) El Hassan IM, Sahly A, Alzahrani MH, Alhakeem RF, Alhelal M, Alhogail A, et al. Progress toward malaria elimination in Jazan Province, Kingdom of Saudi Arabia: 2000-2014. Malar J. 2015; 14(1): 444. doi: 10.1186/s12936-015-0858-1, PMID: 26552387, PMCID: PMC4640236.

15) Al-Jaser MH. Studies on the Epidemiology of Malaria and Visceral Leishmaniasis in Jizan Area, Saudi Arabia. J King Saud Univ. Vol. 19, Science (1), pp. 9-19, Riyadh (1427H./2006)

16) Lara AM, Kandulu J, Chisuwo L, Kashoti A, Mundy C, Bates I. Laboratory costs of a hospital- based blood transfusion service in Malawi. J Clin Pathol. 2007; 60: 1117-20. doi: 10.1136/jcp.2006.042309, PMID: 17412875, PMCID: PMC2014856.

17) Allain PJ. Malaria and Transfusion: A Neglected Subject Coming Back to the Forefront. Infect Dis. 2010; 51(10): 1199-200.

18) Rosenberg R, Wirtz RA, Schneider I, Burge R. An estimation of the number of malaria sporozoites ejected by a feeding mosquito. Trans R Soc Trop Med Hyg. 1990; 84: 209-12. doi: 10.1016/0035-9203(90)90258$\mathrm{G}$

19) Jamjoom MB, Ashankyty IM, Azhar EA, Tonkol AK, Al-Harthi SA. Detection of malaria in Saudi Arabia by real-time PCR. J Egypt Soc Parasitol. 2006; 36(3): 737-48. PMID: 17153692

20) Al-Tawfiq JA. Epidemiology of travel-related malaria in a non-malarious area in Saudi Arabia. Saudi Med J. 2006; 27(1): 86-9. PMID: 16432601.

21) Alkhalife IS. Imported malaria infections diagnosed at the Malaria Referral Laboratory in Riyadh, Saudi Arabia. Saudi Med J. 2003; 24(10): 1068-72. PMID: 14578970.

22) Coleman M, Al-Zahrani MH, Coleman M, Hemingway J, Omar A, Stanton MC, et al. A country on the verge of malaria elimination--the Kingdom of Saudi Arabia. PLoS One. 2014; 9(9): e105980. doi: 10.1371/journal.pone.0105980, PMID: 25250619, PMCID: PMC4175080.

23) Kitchen AD, Chiodini PL. Malaria and blood transfusion. Vox Sang. 2006; 90: 77-84. doi: 10.1111/j.14230410.2006.00733.x, PMID: 16430664.

24) Moody A. Rapid diagnostic tests for malaria parasites. Clin Microbiol Rev. 2002; 15: 66-78. doi: 10.1128/CMR.15.1.66-78.2002, PMID: 11781267, PMCID: PMC118060.

25) Milne LM, Chiodini PL, Warhurst DC. Accuracy of routine laboratory diagnosis of malaria in the United Kingdom. J Clin Pathol. 1994; 47: 740-2. doi: 10.1136/jcp.47.8.740, PMID: 7962629, PMCID: PMC502149.

26) Benito A, Rubio JM. Useful of seminested polymerase chain reaction for screening blood donors at risk for malaria in Spain. Emerg Infect Dis. 2001; 7: 1068. doi: 10.3201/eid0706.010632, PMID: 11747747, PMCID: PMC2631925.

27) Kitchen AD, Barbara JAJ, Hewitt PE. Documented cases of post transfusion malaria in England: a review in relation to current and proposed donor-selection guidelines. Vox Sang. 2005; 89: 77-80. doi: 10.1111/j.1423-0410.2005.00661.x, PMID: 16101687.

28) Nansseu JR, Noubiap JJ, Ndoula ST, Zeh AF, Monamele CG. What is the best strategy for the prevention of transfusion-transmitted malaria in sub-Saharan African countries where malaria is endemic? Malar J. 2013; 12: 465. doi: 10.1186/1475-2875-12-465, PMID: 24373501, PMCID: PMC3877868.

29) Kitchen AD, Lowe PHJ, Lalloo K, Chiodini PL. Evaluation of a malarial antibody assay for use in the screening of blood and tissue products for clinical use. Vox Sang. 2004; 87: 150-5. doi: 10.1111/j.14230410.2004.00561.x, PMID: 15569066. 
30) Shehata N, Kohli M, Detsky A. The cost-effectiveness of screening blood donors for malaria by PCR. Transfusion. 2004; 44: 217-28. doi: 10.1111/j.1537-2995.2004.00644.x, PMID: 14962313.

31) Saeed AA, Al Rasheed AM, Al Nasser I, Al Onaizi M, Al Kahtani S, Dubois L. Malaria screening of blood donors in Saudi Arabia. Ann Saudi Med. 2002; 22(5-6): 329-32. PMID: 17146254.

32) Alkhunaizi AM, Al-Tawfiq JA, Al-Shawaf MH. Transfusion-transmitted malaria in a kidney transplant recipient. How safe is our blood transfusion? Saudi Med J. 2008; 29(2): 293-5. PMID: 18246245.

33) Slinger R, Giulivi A, Bodice-Collins M, Hindieh F, St John F, Sher G, et al. Transfusion-transmitted malaria in Canada. Can Med Assoc J. 2001; 164: 377-9 